\title{
Erratum to: Distribution angle control of a light-emitting diode downlight lens with high color uniformity using a scattering polymer
}

Keiichi Mochizuki $^{1,2} \cdot$ Kazumasa Oosumi $^{1} \cdot$ Fumiaki Koizumi $^{1} \cdot$ Yoshinori Shinohara $^{1}$. Akihiro Tagaya $^{2} \cdot$ Yasuhiro Koike $^{2}$

Published online: 5 August 2015

(C) The Optical Society of Japan 2015

Erratum to: Opt Rev (2015) 22:422-426

DOI 10.1007/s10043-015-0085-6

The first affiliation should be shown as "Nittoh Kogaku

K.K.", not "Nittho Kogaku K.K.”.

The correct affiliation is as follows.

Nittoh Kogaku K.K., Suwa, Nagano 392-0021, Japan

The online version of the original article can be found under doi:10.1007/s10043-015-0085-6.

Keiichi Mochizuki

kmochizuki@nittohkogaku.co.jp

1 Nittoh Kogaku K.K., Suwa, Nagano 392-0021, Japan

2 Keio Photonics Research Institute, Keio University,

Kawasaki, Kanagawa 212-0032, Japan 